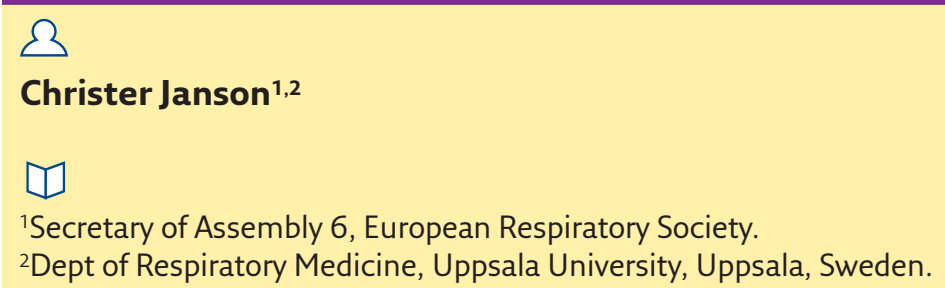

15ecretary of Assembly 6, European Respiratory Society

${ }^{2}$ Dept of Respiratory Medicine, Uppsala University, Uppsala, Sweden.

\title{
Assembly 6: occupation and epidemiology
}

\section{Meet the Assemblies}

Cite as: Janson C. Assembly 6 occupation and epidemiology. Breathe 2016; 12: 281-282.
Assembly 6 is an interdisciplinary assembly that gathers together pneumologists, epidemiologists, clinicians, statisticians, occupational doctors, air pollution scientists and health educators. The assembly now has almost 500 members and has seen a steady growth in membership. Assembly 6 comprises four different groups that complement each other and often work together in joint activities. The groups are: 6.1) epidemiology; 6.2) occupational and environmental health; 6.3) tobacco, smoking control and health education; and 6.4) genes and environment.

\section{1) Epidemiology}

The epidemiology group has about 150 members. The main focus of the group is to understand the natural history of asthma, chronic obstructive pulmonary disease (COPD) and other pulmonary diseases, thereby finding means for risk assessment and primary and secondary prevention. Many of the members work together in research networks created around large international epidemiological studies such as the European Community Respiratory Health Survey (ECRHS) and the Burden of Obstructive
Lung Disease (BOLD) study as well as large national studies like Obstructive Lung Disease in Northern Sweden (OLIN) and the Swiss study on Air Pollution and Lung Disease in adults (SAPALDIA). The Ageing Lungs in European Cohorts (ALEC) study is a consortium that includes several of these large cohorts. The aim of this consortium is to improve the understanding of risk factors for low lung function, respiratory disability and the development of COPD by using information held within existing cohort studies. Apart from traditional survey tools like questionnaires, the group's members increasingly work with biomarkers, imaging and genetic tools. Investigations where survey data are combined with data from national registries are also becoming more and more common.

\section{2) Occupational and environmental health}

The occupational and environmental health group has about 180 members. The group's main focus is following the evolution of occupational diseases (e.g. asthma, asbestos and agricultural exposures, and other new risks) and integrating occupational 
and environmental diseases into clinical practice. Our main interests include: pneumoconiosis, occupational cancers, asthma and COPD. Currently the group is focusing on the effects of occupational and environmental exposures on respiratory health, and specifically on elucidating the mechanisms for air pollution-induced airway inflammation, as well as performing risk assessments. The group also wishes to improve the training of pulmonary physicians in the occupational aspects of respiratory disease.

\section{3) Tobacco, smoking control and health education}

The tobacco, smoking control and health education group has around 150 members. The group works to promote a proactive role for all European respiratory physicians in tobacco control activities, and works together with other ERS groups and bodies to integrate smoking status assessment and smoking cessation therapy into current management of a patient's respiratory condition. The group is working to increase smoking cessation knowledge and skills among all chest physicians and to facilitate research investigating the characteristics of smokers with respiratory disorders and new treatments to quit smoking.

\section{4) Genes and environment}

Genes and environment is the youngest group in our assembly. The aim of the working group is to facilitate studies of gene-environment interactions in asthma, COPD and other respiratory diseases by bringing together the different disciplines involved, thereby identifying new major pathways underlying respiratory diseases. The group welcomes members with a broad range of expertise and interest from within all assemblies of the ERS.

\section{Activities}

The programme for the annual ERS International Congress is one of the main focuses of our assembly. In 2015, more than 400 abstracts were presented from our assembly. We were responsible for six symposia, three postgraduate courses and several other activities. Examples of such activities in 2015 were a postgraduate course on "Gene-environment interactions in lung disease" focusing on understanding gene-environment interactions and the different exposures that occur at different stages of life and a symposium on "Air pollution health effects: from epidemiology to controlled human exposure". The assembly is also involved in several task forces including the recently published "Statement on smoking cessation in COPD and other pulmonary diseases and in smokers with comorbidities who find it difficult to quit" [1] and the ongoing task force on the occupational burden of respiratory disease.

\section{The future}

Our assembly is increasingly engaged in investigating the health-related effects of many of the issues challenging society today such as global warming, globalisation and immigration. Our research will be facilitated by the rapid technological progress in analysing biomarkers, genetics and epigenetics, and handling of big data. The assembly has seen a steady growth in membership and we look forward to engaging an even larger number of participants in the scientific and educational activities of our assembly in the future. 